\title{
Positive relationship between stress and periodontal disease?
}

\author{
Abstracted from \\ Peruzzo DC, Benatti BB, Ambrosano GM, et al. \\ A systematic review of stress and psychological factors as possible risk factors for periodontal disease. \\ J Periodontol 2007; 78: 1491-1504 \\ Address for correspondence: Daiane C Peruzzo, Division of Periodontics, School of Dentistry at Piracicaba, \\ State University of Campinas, Piracicaba, SP, Brazil
}

\section{Question: Is psychological stress a risk factor for periodontal disease?}

\begin{abstract}
Data sources Medline and the Cochrane Oral Health Group Specialist Trials Register were utilised to find relevant studies.

Study selection Articles were examined independently by two reviewers, and all review articles and animal studies were excluded. Studies published in the English language were included if they controlled for the potential effect of confounding factors, had adequate criteria to define periodontal disease, and it was possible to establish evaluate stress levels and the methodological quality of the study. Data extraction and synthesis Data were extracted by two reviewers independently and verified by a third. A qualitative summary of the findings was presented.

Results One prospective clinical trial, seven case-control studies and six cross-sectional studies were included. Eight studies found a positive outcome between psychosocial factors or stress and periodontal disease; four studies observed a positive outcome for some characteristics and a negative outcome for others; whereas two studies found a negative outcome between psychosocial factors or stress and periodontal disease. Conclusions Within the limitations of this systematic review, the majority of studies showed a positive relationship between stress or psychological factors and periodontal disease. In the future, however, well-designed and more representative studies should be considered to confirm these factors as a risk for periodontal disease.
\end{abstract}

\section{Commentary}

The concept that psychological state and, specifically, heightened levels of stress have an impact upon the process and progress of any disease is not new. The authors here cite evidence that this idea has been considered by periodontal researchers at least as long ago as the 1950s. With this in mind, it is certainly appropriate to attempt to consolidate the combined knowledge on this topic, here, by the process of a systematic review.

The authors limited their search to publications from between January 1990 and April 2006 that were identified in two databases, along with a search of the reference lists of the original and review articles that were found. Of the 58 articles identified for review, 21 of the abstracts were considered appropriate for full article evaluation. Fourteen were then included in the review for analysis, reflecting the rigidly applied search criteria within this systematic review framework. This method of bringing together related research is very powerful, but it should be remembered what the exact focus of the question was, "is there sufficient evidence to suggest that stress and psychological factors should be considered as a risk factor in periodontal diseases?"

The overall finding of this review was that the majority of studies, eight out of 14 , showed a positive relationship between psychological factors and stress with periodontal diseases. Two of them were in complete opposition to the concept, however. The complexity was acknowledged by the authors, of the questions asked in the original articles: there is extensive confounding when evaluating causality in periodontal diseases. It was also recognised that there are difficulties in combining or aligning data where different criteria have been used to determine periodontal disease or health, psychological health and the assessment of levels of stress. The authors suggest that this may explain the lack of agreement between the identified studies and ask, of the underlying mechanism, why there is an increased risk in these groups. Was it that the underlying psychological factors were altering the reaction of the host at a purely physiological level, thus, for example, impacting on the pathways of inflammation? Alternatively, does the stressed individual have behavioural traits that put them more at risk, perhaps reducing the desire or ability to undertake effective plaque removal? Further to this, how would these potential mechanisms interact?

The challenge now for the periodontology community is to attempt to unpick the elements identified in the reviewed studies, not forgetting to extend the search laterally and ensure there is no loss of previous work excluded by the systematic review process. By doing this, it may become possible to design future interventions to evaluate the impact of psychological factors and stress - in more quantifiable terms - upon the initiation, progress and management of periodontal diseases.

\section{Practice point}

- This review provides further evidence for multifactorial drivers of periodontal diseases. It is vital to remember the underlying principles of managing people who have any disease: consideration and management of the underlying causes is imperative. Within the plethora of risks associated with periodontal destruction, stress remains a credible but poorly understood and quantified element.

Giles McCracken School of Dental Sciences, Newcastle University, Newcastle upon Tyne, UK.

Evidence-Based Dentistry (2009) 10, 42. doi:10.1038/sj.ebd.6400645 